\title{
Medición del valor del cliente
}

\section{Customer value measurement}

\author{
Humberto Serna Gómez \\ Fundación Universitaria María Cano, Medellín, Colombia. \\ Alejandro Díaz Peláez \\ Institución Universitaria Pascual Bravo, Medellín, Colombia.
}

\section{Resumen}

Hoy día, medir el valor para los accionistas es una práctica incorporada en el mundo de la valoración de las empresas. Estas valoraciones generalmente no incluyen al cliente como un activo generador de valor, por lo que desde el marketing se propone una visión en la que el cliente es concebido como un activo intangible de la empresa, un agente generador de valor que se construye y desarrolla durante su ciclo de vida, a través de un proceso de relaciones perdurables en un periodo de tiempo determinado. En tal sentido, el Valor Presente Neto del cliente como línea de base, permite predecir el valor del cliente, además facilita la definición de las estrategias comerciales y de mercado y por ende para que su ciclo de vida sea generador de valor tanto como para el cliente como para la empresa. Así, el cálculo del valor del cliente durante el ciclo de vida de un contrato o compromiso, respaldado por medidas como la atracción, retención, profundidad de penetración del producto y/o servicio, satisfacción o número de referencias integra herramientas de evaluación que permiten a una empresa tener una mayor comprensión del valor generado por su inversión en el cliente como un verdadero activo de la organización. Palabras clave: Activo empresarial, estrategia de mercadeo, fidelización de clientes, gestión comercial, valoración de empresas.

Clasificación JEL: M310

\section{Abstract}

Autor de Correspondencia:

a.diazpe@pascualbravo.edu.co

Recibido: 10-09-2019

Aceptado: 07-02-2020

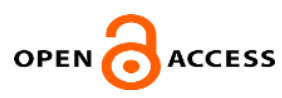

(cc) BY

Copyright (C) 2020

Desarrollo Gerencial
Today, measuring the value for shareholders is a practice incorporated into the world of business valuation. These valuations generally do not include the customer as a value-generating asset, so marketing proposes a vision in which the customer is conceived as an intangible asset of the company, a value-generating agent whose value is built and developed during its life cycle through a process of enduring relationships over a given period of time. In this sense, the Net Value of the customer as a baseline allows the prediction of the customer's value and also facilitates the definition of commercial and market strategies for its life cycle to be a generator of value both for the customer and company. This, the calculation of customer value during the life cycle of a contract or commitment, supported by measures such as attraction, retention, depth of product and/or service penetration, satisfaction, or number of references integrates assessment tools that allow a company to have a better understanding of the value generated by its investment in the customer as a true asset of the organization.

Keywords: Corporate assets, marketing strategy, customer loyalty, commercial management, business valuation. JEL Classification: M310

Como citar este artículo (APA):

Serna, H. y Peláez, A. (2020). Medición del valor del cliente. Desarrollo Gerencial, 12(1), 1-26. DOI: https://doi.org/10.17081/dege...3356 


\section{Introducción}

Este documento presenta una revisión evaluativa acerca del Valor del Cliente. Este concepto ha venido evolucionando en los últimos años y se ha convertido en un activo intangible. La perspectiva de medir al cliente como un activo en el que se invierten recursos, es un cambio de visión que abre un nuevo camino en el mundo empresarial; el problema radica realmente en identificar las perspectivas que se pueden usar para hacer esta medición. Hasta la actualidad se han empleado métodos financieros como la tasa de retención de los clientes, costo de adquisición de un cliente, la tasa de conversión, el ticket promedio de compra y la tasa de rotación de clientes.

Teniendo en cuenta lo anterior, medir el Valor del Cliente como un activo (Customer Equity), fue el propósito fundamental de este trabajo; para lo cual primeramente se realizó una revisión teórica, posterior a ello, se procedió a realizar un análisis general del concepto valor en el cual se introdujeron los conceptos de valor para el cliente y ciclo de vida del cliente, y por último un análisis de las perspectivas para realizar esta medición.

Por otro lado, se propone también medir el Valor Presente Neto del Cliente, a través del Customer Lifetime Value -CLV que está direccionado por la información de los clientes a partir del Customer Relationship Management-CRM y, en otros casos, con probabilidad de ocurrencia de eventos, hasta usar la valoración desde la óptica del cálculo actuarial.

Los aportes de este trabajo están en la profundización de los conceptos de valor, del valor del cliente y aportes novedosos sobre el ciclo de vida del cliente.

\section{Fundamentación teórica}

\section{El Concepto Ciclo de Vida del Cliente-CVC}

El CVC es un término usado para describir el desarrollo por el cual pasa un cliente en una organización desde el momento en que adquiere un bien o servicio; para Gupta et ál. (2006), el CVC es "el proceso de adquisición, retención, margen o desarrollo de clientes dentro de una estrategia de mercadeo relacional" (p.140). 
Figura 1

Ciclo de Vida del Cliente

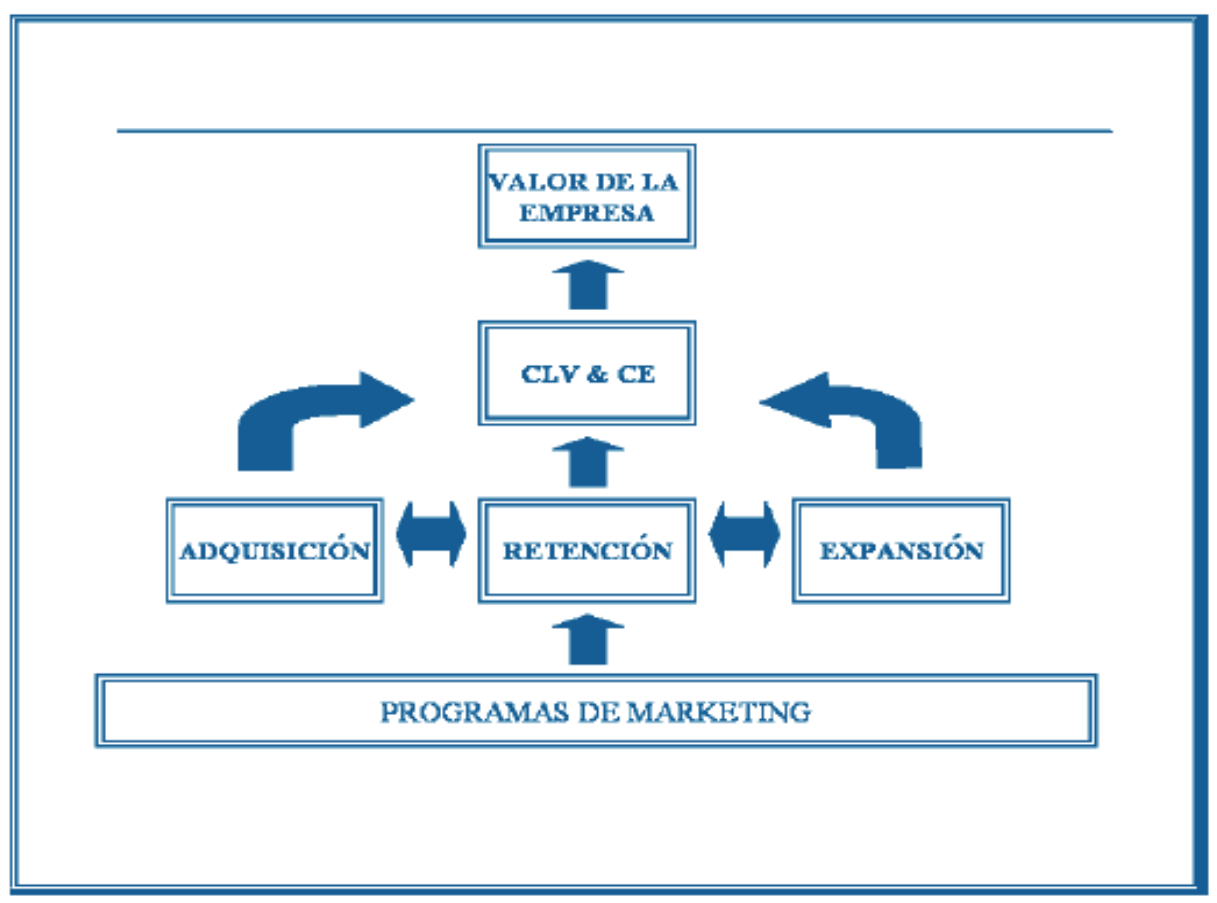

Fuente: Gupta et ál. (2006).

Por su parte, Trajecta, citado por García (2008) afirma que:

El Customer Relationship Management (CRM) permite a la empresa conocer al cliente a través de su ciclo de vida (...) Los clientes a lo largo de su relación con la empresa pasan por distintos estadios, el conocimiento de estos y saber en cada momento en cuál de ellos se encuentran posicionados los clientes es uno de los objetivos principales de una estrategia del CRM. (p. 28)

En el concepto de Ciclo de Vida del Cliente-CVC existen varios elementos fundamentales para definir las estrategias de marketing y la desagregación de las etapas del mercado; sin embargo, cuando se construye del ciclo de vida, el conocimiento del cliente es esencial y para esto es necesario contar con una base de datos confiable que sea la fuente para la toma de decisiones frente a la relación con el cliente; en tal sentido, la gestión de datos, es un elemento fundamental para medir el valor del ciclo de vida del cliente (Figura 2). 
Figura 2

Gestión de Vida del Cliente

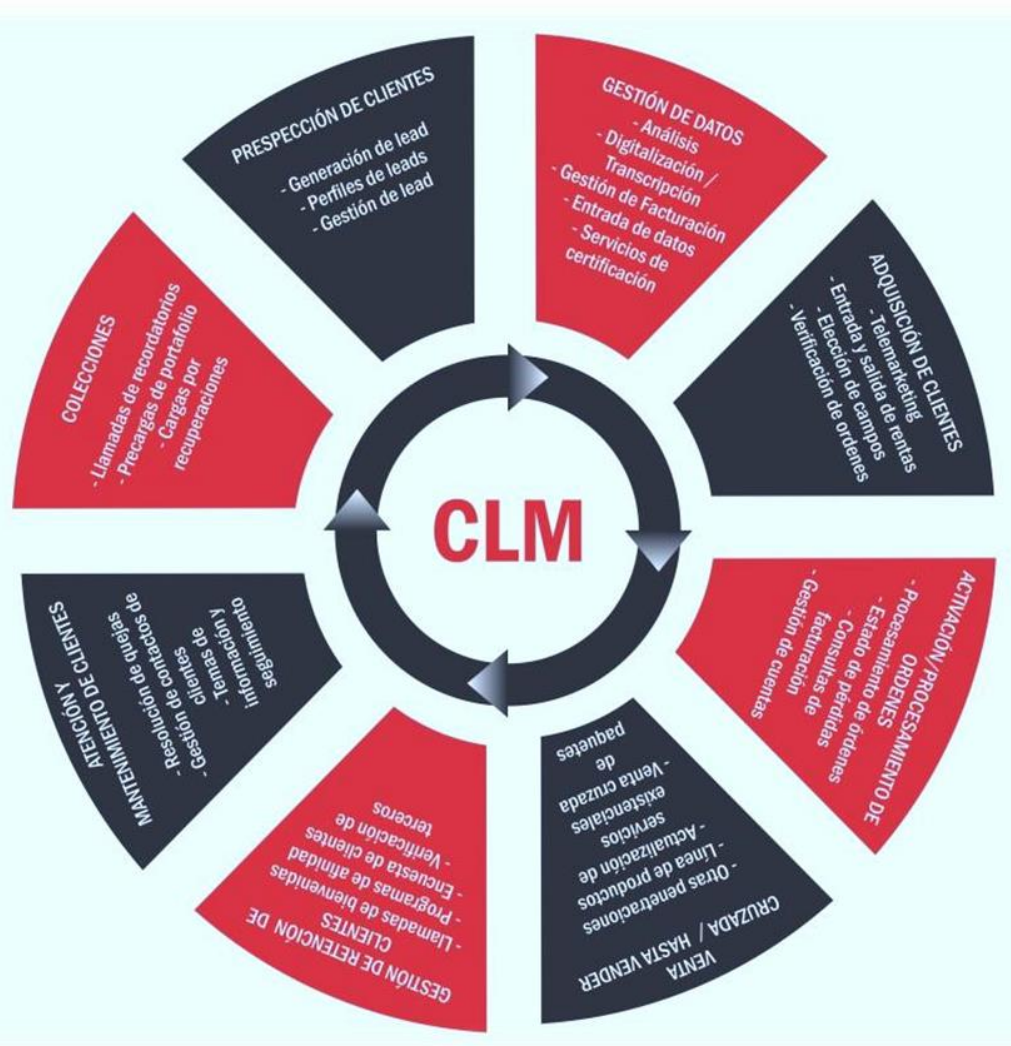

Fuente: Pons (2012)

De acuerdo con Pons (2012):

El Customer Lifetime Management-CLM, engloba un concepto más amplio ya que permite recopilar la máxima información de un cliente, mientras que el CRM cuida la relación actual con el cliente, decir, estudia todo el ciclo de vida en relación a la empresa permitiendo considerar de manera más analítica la evolución desde que era un potencial cliente hasta el día de hoy. (párr. 1)

Por otra parte, Avery, Fournier y Wittenbraker (2014) señalan que muchas compañías aún no entienden el significado y la profundidad implícita que hay en las relaciones con los clientes y proponen que es necesario que las empresas desarrollen como competencia, la inteligencia relacional, que sean conscientes de cuáles y cuántas son las relaciones que generan valor para un determinado segmento de clientes y cómo deben potenciarlas.

Además, estos autores sostienen que no basta solo con tener la información demográfica relacionada con la edad, el estado civil, los ingresos, el nivel educativo y la frecuencia de compra, sino que también es necesario ir más allá de eso, convertir el relacionamiento en un proceso de aprendizaje dinámico y 


\section{Humberto Serna Gómez, Alejandro Díaz Peláez}

sistémico entre la empresa y el cliente; luego entonces, aprendizaje y conocimiento son los pilares de la nueva era del relacionamiento entre los clientes y la empresa.

De esta manera se puede definir El Ciclo de Vida del Cliente - CVC, como el comportamiento que tiene un cliente a través de los procesos de relacionamiento durante su vinculación con la empresa en la cual ocurren el contacto, transacciones, interacciones, momentos de verdad, recompras, entre otros, que son eventos que generan experiencias y aprendizajes que la organización debe capitalizar con su inteligencia relacional, para hacer que las relaciones sean generadoras de valor compartido para el cliente y la empresa. Sin embargo, hay que precisar, que el ciclo de vida no es un proceso simple, están en juego demasiadas variables; esto induce, a desarrollar un modelo sistémico que establezca el Ciclo de Vida del Cliente en cuatro procesos o etapas: Planeación, Adquisición, Desarrollo y Disposición del Cliente.

Figura 3

Ciclo de Vida del Cliente-CVC

\section{CICLO DE VIDA DEL CLIENTE}

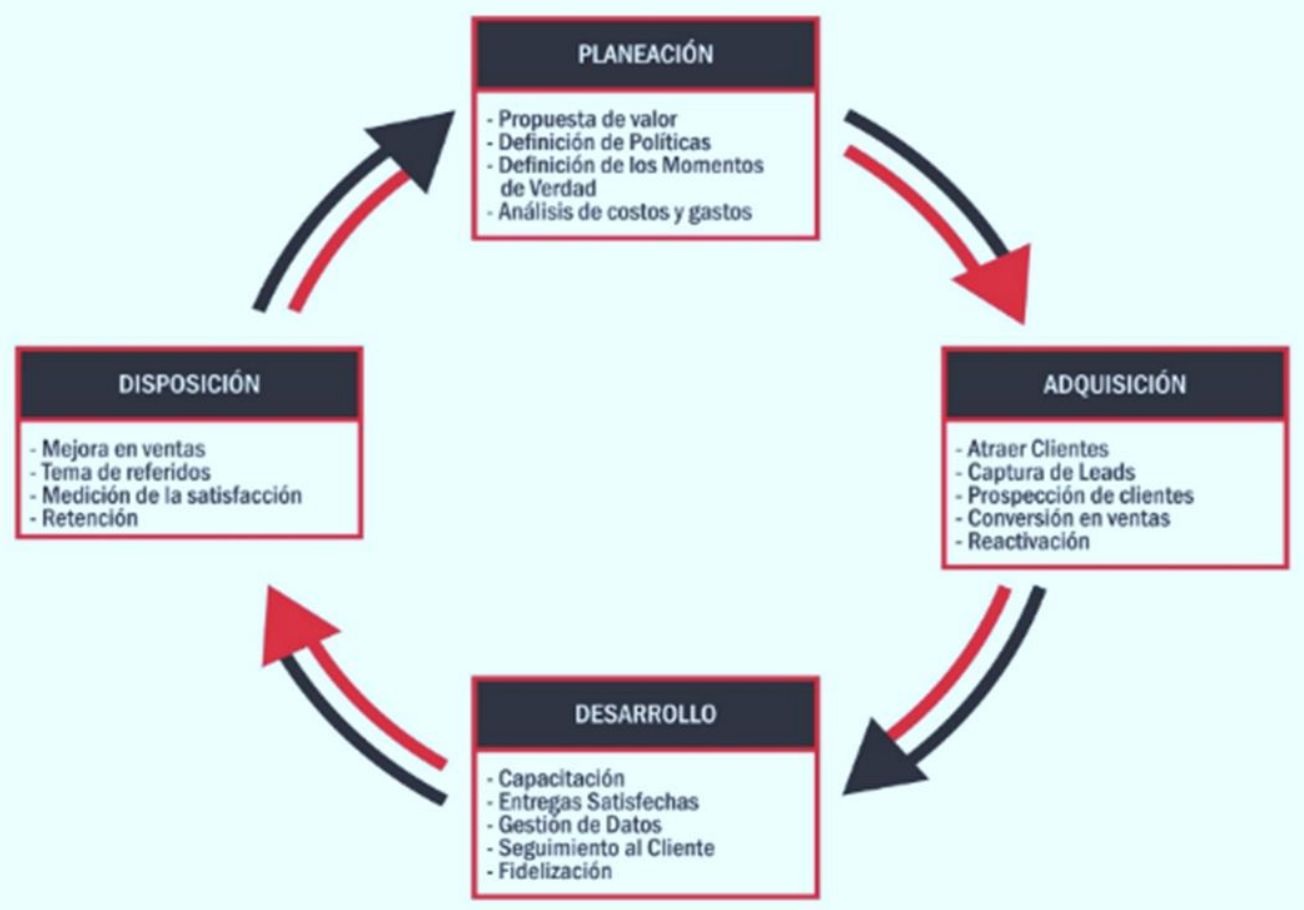

Fuente: Elaboración propia (2016). 


\section{$>$ Planeación.}

En esta etapa se realizan todos los planes en la organización para atraer al cliente. Tanto la empresa como el cliente, invierten mucho tiempo, mientras la empresa desarrolla estrategias de acercamiento a ese cliente potencial, éste explora sus expectativas. Esta etapa, procura definir la propuesta o promesa de valor, las políticas de mercadeo; identificar los momentos de verdad para establecer mediante estrategias cómo responder a ellos y capacitar en tal sentido con el objetivo de lograrlo con cada uno de sus contactos con la organización. Igualmente, analiza y proyecta las inversiones, los costos y gastos relacionados con el CVC.

\section{$>$ Adquisición.}

En la adquisición el cliente conoce el producto o servicio y la propuesta de valor que la empresa le brinda. En esta etapa la empresa diseña las estrategias de mercadeo y comercialización que faciliten su atracción y vinculación; estas pueden ser, mercadeo uno a uno, venta tradicional, venta consultiva, en red, o a través del mercadeo relacional. Aquí se empieza con la identificación del cliente potencial y su vinculación para lo cual requiere una estrategia de mercadeo de captación fundamentalmente transaccional. Debe iniciarse una estrategia de mercadeo de fidelización, cuyo objetivo es hacerle sentir al cliente el valor agregado que le genera su vinculación con la compañía; para logarlo, es necesario desarrollar la atracción de los clientes, la captura de leads, la prospección de Clientes, la conversión en ventas y la reactivación de clientes.

\section{$>$ Desarrollo.}

El cliente ya vinculado, conoce los atributos del producto o servicio, resultado de la estrategia de fidelización; esta etapa es el momento para que la empresa se preocupe porque el cliente realmente disfrute del producto o servicio, conozca a profundidad sus atributos y, sienta placer y deleite en la relación con la empresa. La organización en esta etapa, debe profundizar el conocimiento del cliente, sus gustos, preferencias y hábitos de compra; igualmente, y con el conocimiento que tiene de su cliente, debe empezar a definir y determinar cuál es el portafolio de relaciones que realmente le genera valor al cliente y a la empresa. Al definir este portafolio de relaciones, la inteligencia relacional de la organización, soportada en el conocimiento que ya tiene del cliente, le permitirá diseñar estrategias de mantenimiento, vinculación y de posventa que hagan sentir al cliente como miembro y afiliado a la organización.

La estrategia de fidelización, descrita anteriormente, no puede ser permanente, pues se desgasta, es fácilmente copiable e imitable y con el tiempo puede dejar de ser atractiva. Quedarse con la sola fidelización es muy peligroso, dado que la inversión es muy alta y puede llegar un competidor que la supere, perdiendo así el cliente. Por tanto, la fidelización es una etapa necesaria e importante del 
proceso, pero no se puede quedar allí, hay que transcender hacia una estrategia de mercadeo que convierta al cliente en socio y que asegure la relación a largo plazo. Cuando se escoge la estrategia de mercadeo relacional, se está buscando tener clientes para siempre, construyendo vínculos mucho más fuertes de los que se pretende lograr con la fidelización; el mercadeo relacional, es un enfoque para asegurar una adecuada gerencia de clientes.

\section{$>$ Disposición.}

En esta última etapa, ya el cliente está vinculado a un producto o servicio. Es el momento organizacional para profundizar la fidelidad y empezar un relacionamiento que mantenga la retención y construya confianza como el soporte de una vinculación de largo plazo con la organización. Por ejemplo: programas de postventa que incluyan capacitación en productos y servicios, programas de asociación y membrecía VIP, reconocimientos a la fidelidad, cadenas de referidos, eventos cerrados para clientes especiales, programas de relaciones públicas, son, entre muchas otras alternativas de estrategias para asegurar que clientes sean fieles de por vida. Conocer los niveles de retención, la profundidad de línea en sus compras, la recompras, el índice de referenciamiento e igualmente sus niveles de satisfacción con el servicio que reciben, son indicadores de que la relación de largo plazo se está logrando.

En concordancia, al representar el Ciclo de Vida del Cliente, se pretende comprender el valor del cliente a través de los momentos que él necesita para interactuar con la organización mediante los diferentes procesos, con el fin de maximizar el valor generado; mientras más veces el cliente pase por el ciclo, el retorno de la inversión será más alto. Gracias al ciclo, es posible definir políticas y estrategias para cada proceso y cada paso con el ánimo de lograr satisfacer las necesidades del cliente, con el fin último de mantener un relacionamiento que asegure recuperar la inversión realizada en él. Entendido el ciclo de vida del cliente como un proceso dinámico y sistémico, la organización tiene que definir el proceso y las etapas, anteriormente descritas, que le permitan prepararse y responder a ese ciclo.

En este sentido, para comprender el Ciclo de Vida del Cliente es indispensable conocer el Modelo de Negocio y la estrategia de mercadeo de la empresa, las cuales deben estar alienadas con la filosofía corporativa. Una estrategia de mercadeo relacional, entendida como un proceso sistémico y cíclico, supone un énfasis en las relaciones que ocurren durante el Ciclo de Vida del Cliente quien, al final, es el que genera valor para el cliente y la empresa, además, definir los mercados objetivos teniendo como marco de referencia los ciclos del mercado acordes con los Ciclo de Vida del Cliente.

\section{El Mercadeo}

De acuerdo con lo anterior, desde la perspectiva del mercadeo existe un primer Ciclo Mercadeo de Captación orientado a la incorporación y vinculación de clientes, etapa en la que se enamora al cliente, de tal manera, que se construye una relación emocional sólida. En esta etapa, se debe madurar hacia un 
Humberto Serna Gómez, Alejandro Díaz Peláez

Mercadeo de Fidelización en el cual se busca profundizar la relación, asegurando su fidelización y, por tanto, una profundización en el uso de productos y servicios para luego, llegar a un tercer ciclo de Mercadeo de Relaciones, en la cual se consolida una relación del largo plazo, donde el cliente es un apóstol que predica las bondades de su vinculación con la empresa, que la refiere a otros y atrae nuevos clientes, entre otras posibilidades.

Es en este ciclo de maduración de la relación con el cliente, donde el valor se hace tangible, como se muestra en la Figura 4.

Figura 4

Ciclos del Cliente y el Mercado

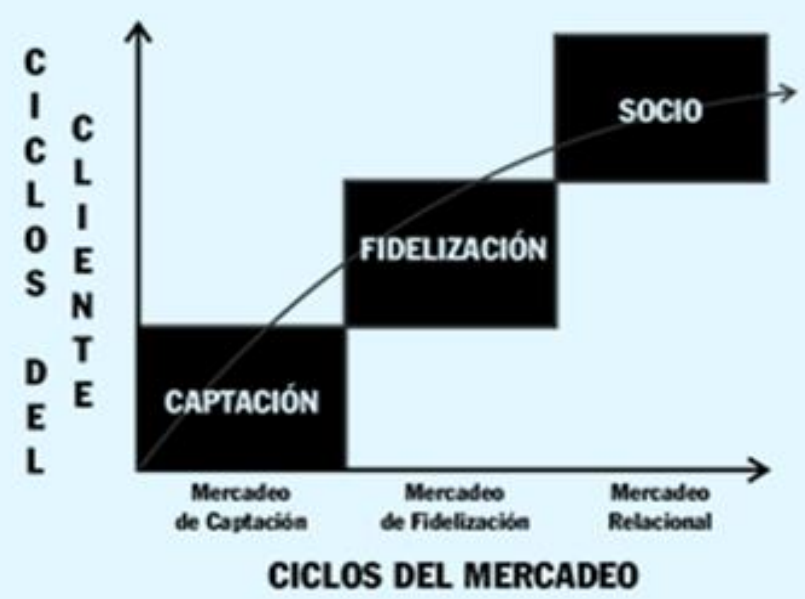

Fuente: Elaboración propia (2016).

\section{Momentos del Mercadeo.}

El valor se construye y se genera en un proceso de relaciones cliente-empresa que requiere tiempo, y supone etapas o momentos de crecimiento que aseguran el valor que genera una relación de largo plazo. Cada momento del mercadeo define una etapa del ciclo de vida para el cliente que requiere de estrategias de mercadeo diferentes o tal como se describe más adelante. 
Figura 5

Momentos del Mercadeo

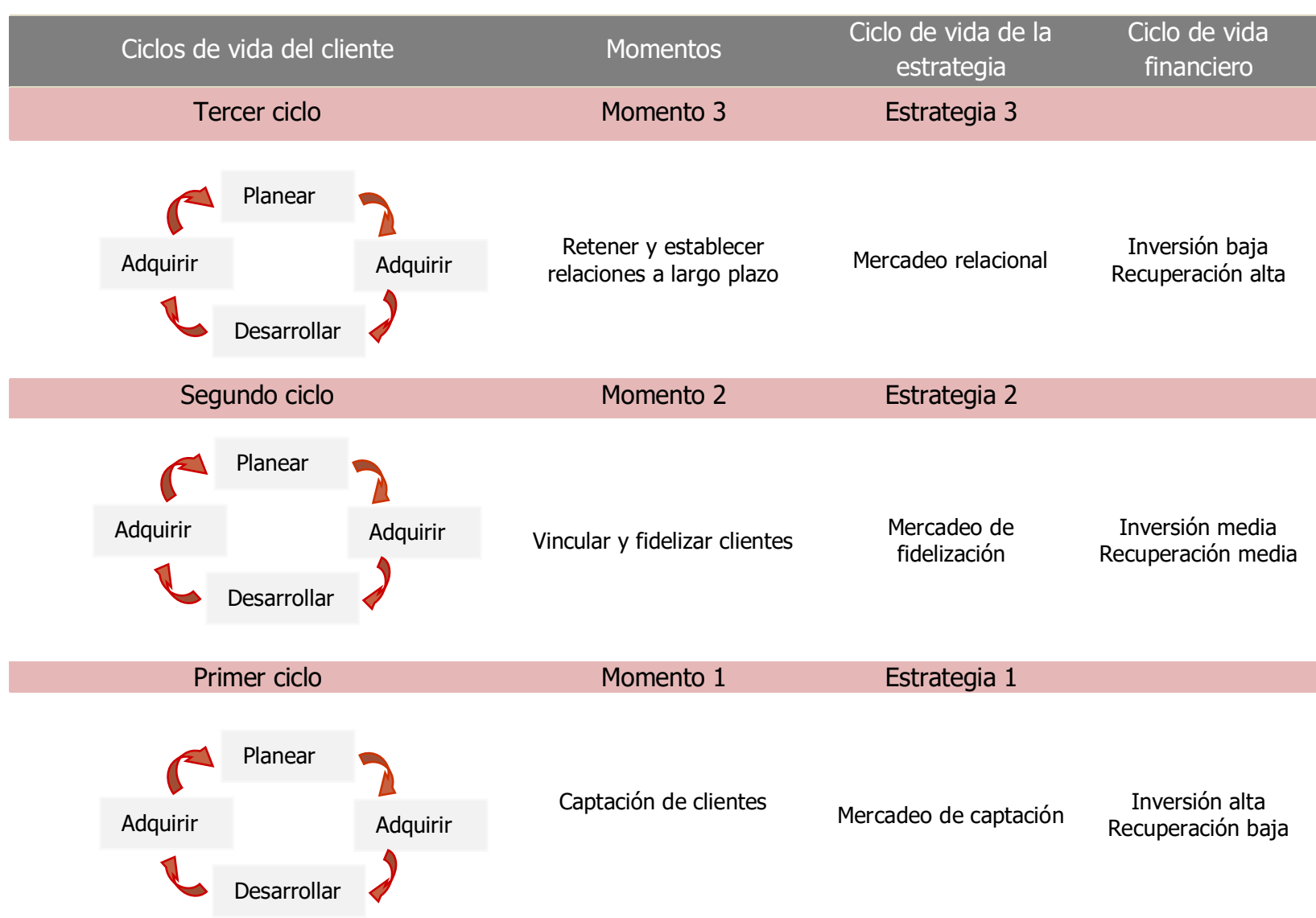

Fuente: Elaboración propia (2016).

De acuerdo con la figura anterior, los momentos del mercadeo presentan las siguientes características:

> Momento 1: Captación de clientes.

Esta etapa de corto plazo se da entre la identificación del cliente potencial y su vinculación con la organización en el cual requiere de una estrategia de mercadeo de captación que fundamentalmente es transaccional.

\section{$>$ Momento 2: Vincular y fidelizar clientes.}

Esta etapa se caracteriza por la vinculación del cliente, inicia con una estrategia de mercadeo de fidelización cuyo objetivo es hacer sentir al cliente el valor agregado que le genera su vinculación con la compañía. Aquí, se requiere un excelente servicio al cliente, así como estrategias de mantenimiento y las de postventa que serán la continuidad en la relación con el cliente. La vinculación del cliente y su afiliación a programas especiales diseñados por la compañía hacen sentir al cliente miembro vinculado y reconocido por la empresa. La fidelización es una etapa necesaria e importante del proceso pero no se 
puede quedar allí. Para ello, se debe trascender hacia una estrategia de mercadeo que convierta al cliente en socio.

\section{Momento 3: Retener y establecer relaciones de largo plazo.}

La estrategia de mercadeo relacional, busca tener clientes para siempre, construir vínculos muchos más fuertes que los que se pretenden lograr con la fidelización. Un cliente apóstol con su voz y su testimonio atrae nuevos clientes (Word Of Mouth). El mercadeo relacional entendido como una estrategia de mercadeo volcada a la construcción de relaciones con el cliente, es el enfoque para asegurar una adecuada gerencia del cliente.

\section{El Ciclo de Valor del Cliente-CVC}

El Ciclo de Valor del Cliente hace referencia al valor que tiene el cliente para la empresa, lo que es diferente al valor que el cliente percibe de la empresa. Para estudiar y medir el valor del cliente, resulta de gran importancia determinar cuáles son las etapas de vida durante su relación con la empresa, esto es lo que se llama Ciclo de Vida del Cliente, el cual permite conocer el volumen, porcentaje de conversión, utilidades y pérdidas en de cada una de estas fases. En este modelo, el CVC busca diseñar y desarrollar estrategias a seguir que permitan generar valor en función del cliente, ya que partir de él se puede identificar qué cuota de mercado se obtiene, la capacidad para dar a conocer la marca, la promesa de valor para captar leads, la capacidad de persuasión para que se conviertan en clientes, el vínculo de los usuarios con la marca, los niveles de retención y profundidad de línea, y así calcular la rentabilidad de su relación para la empresa. Una gestión adecuada de la relaciones del cliente con la empresa, es la fuente de rentabilidad y valor compartido.

En estas definiciones de Ciclo de Vida del Cliente, aparece el concepto relacional como elemento fundamental en la construcción de valor en el corto plazo pero más importante en el largo plazo. Cuando la relación es la generadora de valor, aspectos tan comunes como la medición en mercadeo, la participación en el mercado, volumen de ventas u otras medidas financieras de corto plazo, abren paso otras mediciones tales como la tasa de retención de los clientes, los costos de adquisición o pérdida de clientes y el valor del cliente. Así de esta manera, el Ciclo de Vida del Cliente, permite medir el valor de un consumidor en aquellas empresas que buscan estratégicamente crear relaciones de largo plazo y construir un Customer Equity soportado en la información y conocimiento que se tiene de cada uno de sus clientes, sus hábitos y comportamientos de compra.

Por otra parte, la inteligencia relacional como una competencia organizacional, necesaria para entender las relaciones que realmente generan valor al cliente, evitará saturarlo con estrategias de relacionamiento que al final resultan destructoras de valor. 


\section{Análisis del Concepto de Valor}

Autores como Fragoso (2006) señala que:

Para la escuela neokantiana el valor es una idea, no es concreto, no se encuentra en el mundo sensible y objetivo; es en el pensamiento y es en la mente, donde los valores adquieren forma y valor. Los realistas en cambio, sostienen que los valores son reales, que los valores y los bienes son una misma cosa. (p. 3)

Para Marx (1867), el concepto es entendido como el monto necesario para la producción social de un bien económico, es decir, que tiene valor de uso en un determinado nivel de desarrollo económico; mientras que para Wieser, citado por Brue y Grant (2009), "los factores de producción tienen un valor debido a la utilidad que ellos han conferido al producto final, su utilidad marginal" (p.244).

Por su parte, Anderson citado por Gil y Gallarza (2006), establecieron la diferencia entre valor económico y valor psicológico; para ellas, el valor:

Se "relaciona a" cuando se refiere a que es útil, productivo, posible o comercializable, mientras que cuando se hable del valor psicológico, se dice que es subjetivo y afectivo. Que vale, cuando se "refiere a" la imagen que el sujeto tiene del mismo, y depende de sus sentimientos y afectos (p. 16).

Para Jensen (2008):

El valor es la magnitud subjetiva que se mide por la estima en que el público valore un objeto. El valor está estrechamente ligado al ser humano, sus percepciones y beneficios. Los bienes y servicios tienen valor de una manera cuantitativa en términos financieros, y cualitativos en términos de percepciones. Por lo tanto, los bienes y servicios adquieren valor siempre y cuando el ser humano se los otorgue y reconozca (p. 13)

Se entiende entonces que este concepto de valor en el mundo empresarial, ha sido por muchas décadas como aquel que la empresa genera para sus stakeholders (público de interés) el cual se mide en el mercado y es quien al final es el que define el valor de la empresa.

De acuerdo con Vargo y Lusch (2008):

El valor es siempre único y fenomenológico, está determinado por el beneficiario. El valor es idiosincrásico, experiencial y contextual, y cargado de significado. Esto sugiere que el valor es siempre único y que lo fenomenológico es para determinados beneficiarios, lo significa que cada 
persona tiene una experiencia diferente con el servicio que se intercambia y lo valora de manera diferente (p. 9).

De esta manera, la fijación del valor de uso es esencial para determinar el valor, y el valor de contexto, esto implica a los actores que forman parte de la experiencia y los demás recursos dibujan el valor de la experiencia.

\section{El Concepto del Valor para el Cliente}

Tradicionalmente se ha considerado como valor para el cliente, su satisfacción con el servicio recibido, cuyos atributos fundamentales son: amabilidad, atención, oportunidad y facilidad para obtener el servicio o producto. Moliner, Bigné y Callarisa (2005), señalan al valor para el cliente como: "El valor percibido puede ser considerado la valoración global que hace el consumidor de la utilidad de un producto en base a las percepciones de lo que recibe y lo que da" (p. 164).

Asimismo, Monroe (1990), lo define como "las percepciones de valor del comprador representan el intercambio entre la calidad y los beneficios que ellos perciben en los productos en relación con el sacrificio que ellos perciben al pagar el precio" (p.47).

En la misma línea, Carr (1990), afirma que "valor es el beneficio que un cliente obtiene de su producto o servicio, menos el coste que le supone comprarlo" (p.167), lo que concuerda con Lovelock (1991) al señalar que "el valor neto es la suma de todos los beneficios percibidos, menos la suma de todos los costes percibidos" (p. 82).

Por otro lado, Anderson, Chain y Chintagunta (1993), aproximan su concepto a los mercados al expresar que "el valor en los mercados de negocios es el valor percibido en unidades monetarias, entendidas estas como los sacrificios económicos, técnicos, de servicios y sociales recibidos por la organización cliente, de un precio pagado por el producto" (p. 3).

Desde otro ángulo, Gale (1994), define el valor en términos del valor del cliente al sostener que "el valor del cliente es la calidad percibida en el mercado ajustada al precio relativo en el mercado" (p.50). Para Kotler, Cámara y Grande (1995), "el valor supone la estimación por parte del consumidor de la capacidad de los productos para satisfacer sus necesidades" (p. 140).

Con una óptica muy similar, Goodstein y Butz (1996), aseguran que "el valor para el consumidor es el vínculo emocional establecido entre el productor y un consumidor después de que el consumidor haya usado el producto o servicio ofertado por el proveedor y encontrado que dicho producto le ha aportado valor añadido" (p. 24). 
Woodruff (1997), asegura que:

El valor para el cliente es una preferencia y evaluación percibida por el cliente de los atributos del producto, de los resultados y de las consecuencias derivadas del uso que facilita alcanzar los objetivos y propósitos del cliente cuando los utiliza. (p. 141)

El concepto de valor para el cliente, también ha sido referido con mayor precisión por Kaplan y Norton (1996) quienes afirman que:

La propuesta de valor para el cliente se compone de un conjunto de elementos que determinan el valor que percibe el comprador; estos, incluyen los atributos del producto, sea un bien o un servicio, la imagen de la empresa y la relación entre la empresa y el cliente. Los atributos comprenden las funciones del producto, su calidad, su precio y el tiempo. (p. 216)

Este último atributo, según Francés (2006), se refiere al "tiempo dedicado por el cliente a la búsqueda del producto, a la espera para que esté disponible, a efectuar la compra y a esperar para que se le cumpla la promesa de valor" (p. 144).

Para Moliner, Bigné y Callarisa (2005), existen tres focos claros de divergencias en las definiciones presentadas:

1) La calidad y satisfacción son términos subjetivos; 2) Las dimensiones positivas como: beneficio esperado, beneficio asumido, la calidad, la satisfacción, la utilidad, el servicio superior, el valor del personal y el valor de la imagen; en las dimensiones negativas, están: el precio, el riesgo, el esfuerzo, los costos tales como la puesta en marcha, operativos, financieros, psíquicos, de disposición entre otros, y 3) El valor percibido difiere en el tiempo que se está considerando dependiendo de las circunstancias que rodeen al cliente. (pp. $96-100$ )

Esto origina que algunos estudiosos no consideren el valor percibido; por esto, los autores que refieren el valor percibido antes del momento de compra, durante y después de ella, llegan a diferenciar el valor deseado y el valor recibido. (Woodruff, 1997, p. 142)

En definitiva, el concepto de valor para el cliente se define como la diferencia entre lo que el cliente paga por un bien o un servicio, y el conjunto de beneficios que recibe. 


\section{Humberto Serna Gómez, Alejandro Díaz Peláez}

Figura 6

Tipos de Valor para el Cliente

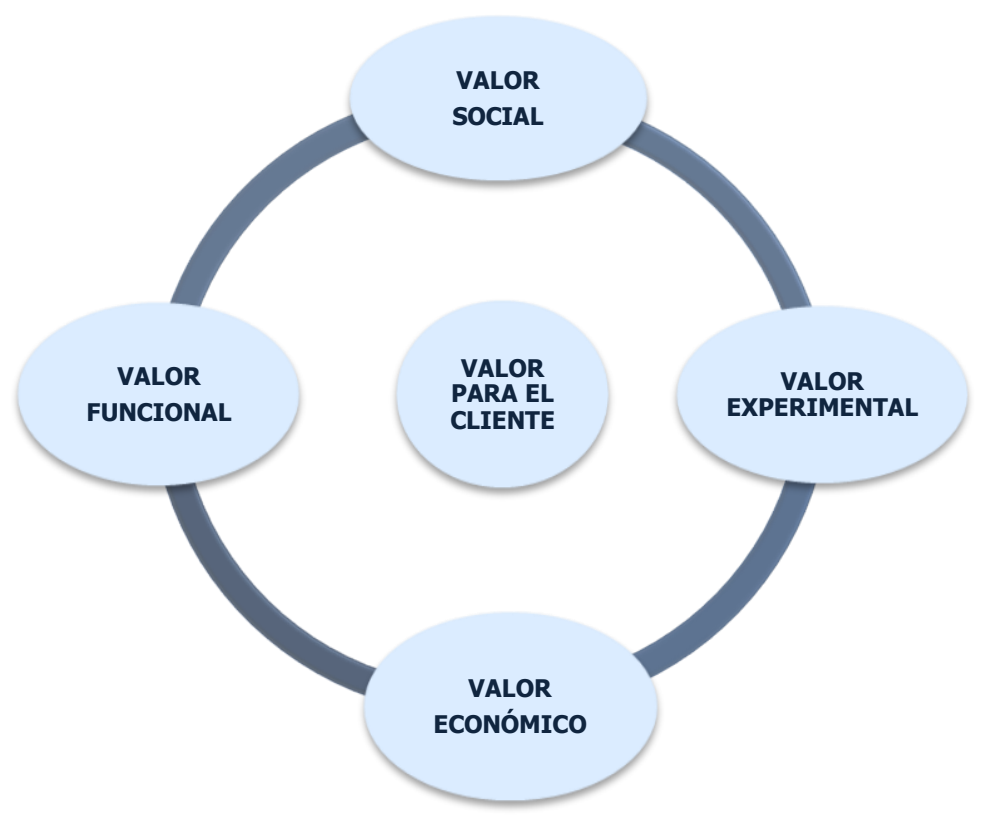

Fuente: Elaboración propia a partir de Gupta (2014).

En este nuevo contexto conceptual, el Valor del Cliente se convierte en un activo empresarial como resultado de un proceso cíclico generado por las relaciones de la empresa con sus consumidores, cuyo valor es posible calcular. Para medir tal valor, es necesario comprender el ciclo de vida durante su vinculación con la empresa, pues es en este proceso donde ocurren las relaciones que generan o destruyen valor tanto para la empresa como para el cliente.

En tal sentido, como lo enuncian Joyanes y Sánchez (2008):

(...) en definitiva, la esencia de la Gestión del Valor del Cliente es predecir, como las acciones de marketing influyen en el CVC. Es estimar el valor de la relación entre el cliente y la empresa en el largo plazo y su efecto sobre el Customer Equity. (p. 66)

En resumen, como bien lo expresan Gupta y Lehmann (2001):

El valor para el cliente depende de los beneficios ofrecidos (desde el punto de vista del cliente) y de los costos involucrados (precio, mantenimiento, servicio). El valor es un concepto muy diferente al costo. Un producto o servicio puede costar muy poco y generar un alto valor. (...) El valor para el cliente incluye dos conceptos de valor a saber: el valor de la categoría, en la cual se asume que no hay competencia y valor relativo (marca) que supone la comparación con otros productos en la misma categoría. (p. 3) 


\section{El Concepto de Medición del Cliente.}

El concepto de valor, está implícito en la promesa de valor que la empresa hace al cliente, es válido en todos los segmentos del mercado, pues el ser humano por naturaleza busca que la satisfacción de sus necesidades y aspiraciones le generen valor, esto implica, romper el paradigma de que solo genera valor el producto o servicio que tiene un precio alto; por consiguiente, todo producto y servicio que satisfaga una necesidad, tiene que generar valor, de lo contrario, es un engaño.

Para Alet (2001):

El valor de la vida de un cliente es definido como el valor actual de los ingresos netos aportados cada año de su vida en la empresa, calculados al tipo de interés considerado como necesario para igualar el coste de capital de la empresa. (p. 27)

En este sentido, las compañías destinan grandes sumas de dinero en programas para el cliente sin poder justificar en términos de valor, cuál es el beneficio que reciben a cambio de estas cuantiosas inversiones. La dificultad en reflejar impactos tangibles sobre los clientes, puede llevar a las compañías a ejecutar estrategias de corto plazo como las promociones que solo muestran resultados rápidos y medibles por un corto tiempo. Por eso, la importancia de medir el valor del cliente se basa en la premisa de que éstos son la principal fuente de ingresos para la compañía. Si se tiene la capacidad de medir el valor de los clientes actuales y futuros de la empresa, entonces se estará muy cerca de calcular el valor de la firma dado que los clientes son activos; todo lo que se consideraba como gasto de mercadeo para adquirir y retener clientes, pasa a considerarse como una inversión. De tal manera que, enfocar la organización a mantener una relación transaccional con el cliente, es tener una visión de beneficios a corto plazo; por el contrario, la orientación a mantener una relación de largo plazo con el cliente, genera utilidades y ganancias mayores.

Autores como Martínez, Borja y Carvajal (1999), señalan que los clientes han de ser considerados como un activo (Consumer Equity) más de la empresa, ya que ellos son generadores de flujo continuo de recursos durante todo su ciclo de vida y relación con la organización (p. 64). Por lo tanto, debe se les debe dar igual importancia que a los demás activos e inversiones de la compañía, así como el EVAEconomic Value added-, que se convierte en la metodología que facilita a una empresa medir la generación de valor para sus accionistas.

Según Joyanes y Sánchez (2008, p. 65), para valorar el cliente activo hay que tener en cuenta su valor presente neto; es decir, el valor actual de los flujos netos de caja generados por él a lo largo de su vida y durante su permanencia con la empresa. Según Berger y Nasr (1998), se trata del beneficio o pérdida para la empresa aportado por los clientes, teniendo en cuenta la vida entera de transacción con ella; son 
ganancias o pérdidas que obtiene la empresa de un cliente mientras esté activo en función del costo de atracción, venta y servicio, así como el costo del dinero en el tiempo que fue cliente activo. (p. 23)

De esta forma, cuando una empresa define que el cliente es un activo generador de flujos y rentabilidad en el largo plazo y desea medirlo, generalmente parte del Valor Presente Neto del ClienteVPNC-; es decir, el valor actual de los flujos netos de caja generados por el cliente, a lo largo de su vida y durante su permanencia en la empresa. A este concepto de valor del cliente, se le denomina Customer Life Time Value o Valor de la Vida del Cliente -CLV. Como lo describen Berger y Nasr (1998), el CLV es el beneficio o las ganancias que obtiene la compañía en la gestión de sus clientes, mientras esté activo en su relación con la empresa e indica el margen bruto que deja cada cliente mientras es fiel a la empresa. (p. 18)

Los resultados del CLV deben de ser complementados con mediciones periódicas de los niveles de satisfacción de los clientes, así como el uso del NPS (Net Promotor Score) para generar relaciones de estos con sus grupos de referencia, usando escalas Likert. Recuérdese que el CRM capta su información cuando pasa su tarjeta de cliente fiel y elabora su perfil como comprador (que compra, frecuencia, cuánto gasta por categoría, etc.). Esta técnica se podría aplicar por clientes individuales, pero para este caso se requieren técnicas econométricas avanzadas, como en el caso de negocios con miles de tiendas y millones de clientes.

Ortigosa (2006), afirma que además de las mediciones tradicionales como participación en el mercado, volumen de ventas y medidas financieras a corto plazo, se abren campo a nuevos indicadores como la tasa de retención de clientes, el costo de adquisición de un nuevo cliente, el valor del ciclo de vida de cliente entre, entre otros (p.3). Esto coincide con lo que afirma Gilibets (2013), quien señala que se debería lograr la objetividad para encontrar criterios de cálculo comparando el valor con el mercado, la competencia, el entorno, para lo cual se deben adoptar criterios como el Valor del Ciclo de Vida del Cliente, el Costo de Adquisición del Cliente, la Tasa de Conversión, el Ticket promedio de compra y la Tasa de rotación de los clientes (Churn). Aparte, es necesario realizar un control de las pérdidas y un control del ciclo. Para Pons (2014), deben incorporarse otros elementos al realizar las mediciones de valor, como son la estrategia de ventas y el flujo de caja del ciclo de vida del cliente. (p.1)

\section{Flujo de Caja del Ciclo de Vida del Cliente}

Una empresa provee valor al cliente a través de productos y servicios, y un cliente provee valor a la firma a través de las utilidades. Las inversiones que se hagan en un cliente hoy, proveerán los beneficios del futuro; por este motivo, cuando se considera a los clientes como activos, está planteándose implícitamente la necesidad de invertir en ellos. Igualmente, si los clientes son activos y si es necesario invertir en ellos, se requerirá conocer el nivel de retorno de la inversión que se realice dado que no todos 


\section{Humberto Serna Gómez, Alejandro Díaz Peláez}

los clientes proveen el mismo valor; así, que es necesario identificarlos para asignar adecuadamente recursos y plantear, de manera acertada, las estrategias para cada uno de ellos.

Autores como Pons (2014), sugieren que al hacer mediciones sobre el valor del cliente, es importante medir el flujo de caja de su ciclo de vida. A manera de ejemplo la Figura 7, ilustra una medición del Flujo de Caja del Ciclo de Vida un Cliente; éste, inicia con la vinculación de un consumidor a la empresa en una primera fase de incubación y desarrollo del cliente, con unos beneficios y flujos claramente negativos, originados por la alta inversión para lograr su vinculación y unas ventas que crecen lentamente. Las dos primeras fases de incorporación y fidelización, consumen mucha inversión y generan pérdidas que solo se recuperan al final de la segunda fase; es aquí, cuando se comienza a llegar al punto de equilibrio. En consecuencia, las fases tres y cuatro son fundamentales en el retorno sobre la inversión y la generación de valor en la relación con el cliente.

Figura 7

Costo del Ciclo de Vida del Cliente

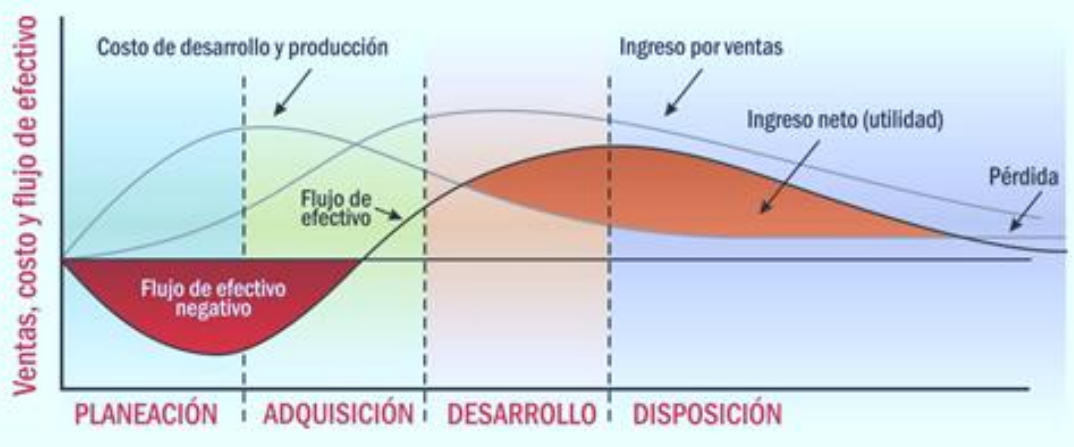

Fuente: Elaboración de los autores (2016).

El punto fundamental de medición del Valor del Ciclo de Vida del Cliente, se basa en la identificación de las etapas de su ciclo de vida y las interacciones y relaciones que ocurren durante el período objeto de medición. De tal manera, que la forma como se defina el ciclo de vida, determinará en gran medida el resultado del valor del cliente. Asimismo, para la medición del valor del ciclo de vida del cliente, la empresa debe considerar cada cliente o grupo de clientes objeto de análisis, como si fuera equivalente a una inversión en un producto o en la participación accionaria en una empresa. Como en toda decisión de inversión con un cliente o grupo de clientes, se puede ganar o perder, así que es factible escoger entre retener o abandonar uno o un grupo de clientes, teniendo como criterio la rentabilidad y el valor que genera. Estas decisiones, son muy importantes y forman parte de las estrategias de mercadeo relacional establecidas por la empresa; lo importante de este enfoque, a diferencia de otros, es que se centra en el cliente como factor de rentabilidad de la empresa, en vez de hacerlo sobre los productos o servicios, práctica tradicional en las empresas que mantienen un enfoque de mercadeo transaccional. 


\section{Cálculo del Valor Presente Neto del Cliente -VPN- (Customer Lifetime Value - CLV).}

La rentabilidad de los clientes, debe ser la reciprocidad de valor que recibe la empresa, la cual debe generar y asegurar la gestión de los clientes y durante el ciclo de su vida con la empresa (Valor Presente Neto -VPN-, o Customer Lifetime Value -CLV-). Durante el Ciclo de Vida del Cliente, se genera una cifra positiva del Valor Presente Neto del Cliente, entendido éste como todo el flujo futuro de utilidad que un cliente produce en la empresa. El valor así determinado, producto de la coproducción en la relación empresa y cliente, tiene dos valores: el valor económico para el cliente y el valor presente neto para la empresa.

El Valor Presente Neto -VPN-, o Customer Lifetime Value -CLV-, es el valor presente de todos los beneficios actuales y futuros del cliente durante su permanencia en la organización. Este concepto involucra dos aspectos: (a) tener en cuenta tanto los beneficios actuales del cliente como también los futuros; y (b), el tiempo que durará el cliente dentro de la organización. De esta manera, el VPN y su rentabilidad, se han convertido en una medida esencial en el mercadeo moderno.

La segmentación por valor del cliente, es el nuevo criterio incorporado en las teorías de mercadeo que otorga nuevas herramientas para la toma de decisiones. El enfoque en el cliente, permite que las estrategias en materia de incorporación, satisfacción, retención y fidelización de los mismos, sean las que generan rentabilidad y valor a la empresa.

Para realizar el cálculo del Valor Presente Neto -VPN-, como primer paso, se requiere el desarrollo de un patrón que refleje el comportamiento del margen que le dará el cliente a la compañía. Este patrón, usualmente presenta un comportamiento negativo al inicio del ciclo debido a los altos costos de adquisición que genera un cliente. A partir del periodo uno, es posible calcular el margen del cliente; la rentabilidad que el cliente le otorga a la compañía, está relacionada con el tiempo de permanencia dentro de la firma. En la medida en que los clientes afianzan su relación con la compañía, los costos de retención disminuyen y el margen se torna cada vez más positivo.

En el siguiente paso, se desarrolla un segundo patrón que refleja cómo sería el comportamiento en un periodo futuro de los clientes que en la actualidad posee la compañía. Los clientes pueden cambiar de proveedor por muchas razones, por ejemplo: cambio de necesidades o preferencias, mejores ofertas de la competencia, u otras; con esta información, pueden hacerse el siguiente análisis: calcular el valor presente neto de las utilidades que genera cada cliente. Aplicar explícitamente esta metodología, puede tornarse difícil por la cantidad de datos, la complejidad y la precisión que se requieren.

Se dice que cuando el Valor Presente de los Activos, tanto tangibles como los intangibles, supera el Valor Presente de los beneficios, existe un superávit actuarial y un déficit actuarial cuando el Valor 
Presente de los beneficios supera el valor presente de estos activos. Para determinar el costo del valor del cliente, se supone el pago de una prima por cliente (que equivale a las utilidades que el cliente genera), para lo cual se estima la probabilidad de que un cliente esté activo al momento de ser calculado. En el caso de deserción de clientes, las tablas de mortalidad constituyen la herramienta estadística que resume la experiencia en tal sentido para la organización; por ello, el desarrollo de dicha herramienta, representa la probabilidad de deserción de un grupo de clientes hasta su extinción, incluso, muestra a diferentes edades la existencia o permanencia del cliente en la empresa. La mortalidad estimada, implica el supuesto de deserción del cliente y como consecuencia, una reducción en las obligaciones para la empresa, lo que representará generar reservas más pequeñas. Por el contrario, si se subestima, dará como resultado una población más grande implicando reservas mayores para que la empresa pueda atender sus clientes, quienes están expuestos a contingencias tales como inconformidades que logren generar deserciones, reclamaciones o rotaciones, los cuales deben ser considerados ya que actúan como fuerzas que disminuyen las obligaciones en inversiones y en mantenimiento de clientes para la empresa.

\section{Método}

Para el desarrollo del presente artículo se realizó una revisión sistemática de documentos orientados a los conceptos: valor para el cliente, medición al cliente, ciclo de vida del cliente y medición del valor del cliente. Asimismo, se consultaron revisiones y estudios científicos sobre el tema tratado.

Para la recolección de la información se localizaron fuentes secundarias en primer nivel en la base de datos EBSCO y SCOPUS, complementados por recursos disponibles en la base de datos Google Académico.

Teniendo en cuenta que el estudio es de carácter documental, los participantes son documentos tipo artículos y libros. De la búsqueda realizada, se seleccionaron 98 documentos referidos a las variables objeto de estudio, constituyendo así la población del presente estudio.

Posteriormente, mediante la aplicación de criterios de exclusión asociados a la cantidad de citas y al material cientifico publicado en revistas indexadas permitió la construcción de una base de datos de 35 documentos que constituyeron la muestra del estudio.

Los datos de las fuentes seleccionadas se extrajeron con base a los relevantes para esta revisión, en especial aquellos orientados a los autores que han venido trabajando el tema en cuestión.

Para el Análisis de la información, ésta fue organizada y analizada en una matriz de estudio cuyo propósito principal fue la identificación de los aspectos relevantes para el tema abordado. 


\section{Interpretación}

Hasta ahora, se manejan tres grupos de indicadores que determinan el valor para la empresa, estos representan a cada cliente o grupo de clientes; el objetivo, es saber cuánto invertir en los clientes de manera que la empresa sea rentable y logre que esto se mantenga en largo plazo.

El primer grupo, está basado en métricas y metodologías para determinar el valor del cliente. Este método fue desarrollado por Gupta y Lehmann (2001), quienes que señalan que es importante "monitorear en forma general e individual el costo y el beneficio de la relación que tiene la compañía con el cliente" (p. 4). El segundo, busca medir el desempeño del cliente en términos de la perspectiva financiera. El tercer y último grupo, corresponde a la adopción de razones financieras para analizar el impacto que están generando los clientes sobre la rentabilidad y las utilidades de la compañía.

Asimismo, para determinar el valor que representa cada cliente para la empresa, es importante establecer cuáles son los más rentables, cuáles representan un margen de bajo a normal y con cuáles está perdiendo dinero. Obtener esta información, aunque parezca obvio, no necesariamente es fácil; esto se debe, a que las empresas organizan sus centros de costos orientados a aspectos diferentes al cliente (el producto, unidades administrativas, etcétera). Por lo tanto, si se desea orientar a la organización a una óptima gestión de clientes, se debe comenzar por obtener la rentabilidad de cada cliente o grupo de clientes representativos. Más aún, si la empresa tiene un sistema de costos-Activity Based Costing (ABC). Sin embargo, obtener estos datos de manera continua no debería ser un inconveniente ya que una vez obtenida esta información, y tal como lo mencionan Kaplan y Narayanan (2001), se pueden tomar acciones para convertir a los clientes no rentables en rentables. Estas acciones, según estos autores, son tres: mejorar los procesos, decisiones sobre precios y manejar las relaciones.

\section{Evaluación critica}

No obstante, el valor no se genera solamente para que la empresa tenga un activo: el cliente; la inversión que hace la empresa en él, tiene que generarle valor $y$, por tanto, es indispensable que el consumidor conozca y comprenda los beneficios que recibe al mantener unas relaciones duraderas con la empresa. Tanto su permanencia, lealtad, profundidad de línea aumentando su participación en el portafolio de la empresa, confianza en la empresa manifestada en el referenciamiento de clientes, como la satisfacción con el servicio que recibe, entre otros, son indicadores de lo que el cliente valora de su relación con la empresa. De esta manera, el valor así concebido, es un proceso de doble vía, generador de valor para el cliente y la empresa, que requiere estrategias de mercadeo centradas en la relación más que en la transacción como lo ha sido en el pasado. Por lo tanto, pasar de un mercadeo transaccional a 
uno relacional, es el gran desafío de las empresas que deciden que sus clientes les generen Valor Económico Agregado-EVAC.

En este sentido, el objetivo a lo largo del tiempo, siempre ha sido en maximizar el valor de la empresa para los stakeholders. Desde la perspectiva financiera, el objetivo ha sido la reducción de costos para lograr mayores beneficios. Pero, ahora bien, desde la perspectiva del marketing, se ha evidenciado que es posible construir una ventaja competitiva a través de la gestión de los clientes soportada en una estrategia de mercadeo relacional.

De acuerdo con lo planteado, la empresa tiene la obligación tanto de generar valor como el deber de asegurar una tasa de retorno de la inversión que hace para entregar valor al cliente. Tal valor, se refleja en el precio que el cliente paga por el bien o servicio, así como en la competitividad y estabilidad que le genera la fidelización y el establecimiento de relaciones de largo plazo con sus clientes. En otras palabras, cuando el cliente reconoce el valor que le genera el producto o servicio que recibe y paga por él. Cuando no hay valor, no hay compra, no hay recompra, no hay referenciamiento, y, en especial, no hay relaciones de largo plazo.

El valor de la empresa ha sido medido tradicionalmente por el valor generado para sus stakeholders. Las inversiones han sido privilegiadas en activos físicos o de infraestructura, que desarrollan capacidades y competencias organizacionales sobre las cuales se construyen las ventajas comparativas y competitivas de la empresa, que al final, generan rentabilidad y valor. En el presente las empresas no se evalúan por sus activos sino por el valor de sus intangibles como clientes o el posicionamiento de las marcas. Por este motivo es necesario establecer una metodología que le permita a una empresa conocer el valor de sus clientes como parte de los activos en la valoración de esta. De esta manera, el cliente como un activo generador de valor para la empresa, incorpora una nueva dimensión en la medición del desempeño de la empresa y su generación de valor tanto para esta como para el cliente.

Por consiguiente, medir el valor del cliente, se convierte en un reto que incorpora un nuevo elemento en la valoración de una empresa. Para esto, se sugiere el uso del método del Valor Presente Neto-VPN-, o Customer Lifetime Value-CLV-. Esta medida, le permite a la compañía tener un valor aproximado de lo que representa para la empresa ese cliente durante el ciclo de vida que lo relaciona al producto o al servicio; en particular, esta medida es útil cuando una empresa decide predecir la rentabilidad y el valor que le generaría, invertir en un segmento de clientes que ya sirve o incursionar en un nuevo segmento de mercado.

Además, estos métodos de valoración, se complementan con otras mediciones conocidas y mencionadas tales como el cálculo del flujo de efectivo del cliente, las tasas de incorporación, retención, frecuencia y profundidad de la compra, referenciamiento, e igualmente con medidas de satisfacción del 
Humberto Serna Gómez, Alejandro Díaz Peláez

servicio que reciben. Por tanto, el valor, se construye con una estrategia de relacionamiento con el cliente que privilegia el largo plazo sobre la relación transitoria o de ocasión.

Por otro lado es relevante mencionar que con las nuevas Tecnologías de la Información-TIC'S aparecen propuestas como la que aporta Kissmetrics, que es una plataforma de analítica web que se caracteriza por la medición en eventos para realizar el seguimiento en tiempo real sobre el comportamiento de los clientes. Esta es una herramienta que sirve para integrar con otras plataformas. Este modelo se utiliza flujos futuros de dinero.

También, se debe importante medir la influencia de los empleados en la satisfacción de los clientes, a traves de la creación de KPI (Key Performance Indicators), Indicadores Claves de Desempeño e indicadores de BSC (Balanced Scorecard-Cuadro de Mando Integral), el cual es una metodología utilizada para definir y hacer seguimiento a la estrategia de una organización.

\section{Contribución del autor}

Teniendo en cuenta todo lo anteriormente descrito, se puede afirmar que el Ciclo de Vida de los Clientes -CVC-, es un concepto que permite medir los eventos en que interviene la vida del cliente en la organización; de tal manera, que podría calcularse aplicando el Cálculo Actuarial como método para valorar el ciclo de vida de los clientes, es decir, que esta metodología se podría utilizar para medir el Valor del Ciclo de Vida del Cliente (Customer Lifetime Value -CLV-).

Asimismo, este modelo puede ser tenido en cuenta para calcular la tasa de retención o esperanza de vida del cliente, representada por la probabilidad de que el cliente se mantenga activo en la organización en los periodos sucesivos. Además, se usa para el cálculo de los fondos de pensiones y de jubilaciones, para estimar la supervivencia de una persona con derecho a pensión; busca proyectar eventos probabilísticos que están sujetos a contingencias y estimar los costos que estos representarán, encontrando las cotizaciones únicas o periódicas para crear los fondos suficientes para hacerles frente.

Por otra parte, el Valor Presente Actuarial, es la valoración de una serie de pagos (inversiones que la empresa realiza en los clientes) en los cuales, además de reconocer que el dinero tiene un valor en el tiempo, estima la posibilidad de que cada pago se haga, razón por lo cual, se le asocia una probabilidad de ocurrencia. El cálculo, se hace por cliente y el resultado total, es la suma de valores presentes netos actuariales de cada uno de las inversiones hechas en cada cliente y que luego estarán al servicio de la empresa. Estos cálculos traen consigo una serie de supuestos empleados sistemáticamente para estimar la probabilidad de ocurrencia de eventos futuros que pudieran generar o evitar el pago de beneficios y así determinar el monto de la obligación contraída; tales supuestos, son fundamentalmente estimaciones ya que se consideran los hechos acontecidos en el pasado y que sirven como guía de lo que probablemente pase en el futuro, hipótesis que contemplan el número de clientes por edad, antigüedad, género, tipo de 
cliente, segmento al que pertenece, número de clientes desertores, o que están inconformes con el producto o servicio, entre otros.

Aparte, es probable que la conducta de los consumidores no sea tan estática y se pueda ver afectada por las promociones, el desarrollo de productos y estrategias digitales, estas variables se deberían considerar cuando se piense usar las técnicas actuariales. Sin embargo, el supuesto fundamental, está en la reserva virtual de capitales y de primas que una empresa hace (que en este caso, es la inversión que la empresa realiza en los clientes), para hacer frente a la captura, mantenimiento y administración de la base de datos de sus clientes.

\section{Conclusiones}

En síntesis, cuando una empresa define su orientación estratégica donde el cliente es un activo, un capital intangible, un generador o destructor de valor, las estrategias e inversiones de mercadeo de la empresa tienen que alinearse con esta definición e incorporar en ellas el relacionamiento como el eje rector de su gestión comercial. Además debe entender no solo las diferentes etapas y momentos del su estrategia de mercadeo, sino también los ciclos por los que pasa su relación con los clientes. Solo así y con este marco de referencia, será posible conocer y medir el ciclo de vida de los clientes y el valor compartido generado durante su relación con ella.

En tal sentido, una efectiva gestión de clientes, debe estar basada en estrategias de conocimiento de los consumidores, así como en la coproducción mediante una relación permanente y transparente, que al final, es la que genera valor compartido para el cliente y para la empresa.

Una empresa que identifica, conoce y valora el ciclo de vida de sus clientes, es quien prioriza las estrategias de retención y de relacionamiento, con objetivos de largo plazo en aquellos clientes o grupos también llamados comunidades, o clúster de clientes generadores de valor. Por ende, entender y administrar el ciclo de vida útil del cliente facilita las decisiones de tamaño y crecimiento, pues no necesariamente el número de clientes se definen por su rentabilidad y valor. De allí, que cuando se piensa en valor, el tamaño debe estar alineado con el modelo de negocio propio de cada empresa.

De esta manera, la introducción de los métodos actuariales, aparecen en este trabajo como una nueva alternativa para la medición del Ciclo de Vida del Cliente, tema que ofrece opciones conceptuales y metodológicas que enriquecen la medición del valor del cliente como un patrimonio intangible de las empresas. 


\section{Referencias}

Alet, J. (2001). Marketing Relacional: Cómo obtener clientes leales y rentables. Barcelona: Gestión 2000.

Anderson, J., Jain, D., \& Chintagunta, P. (1993). Customer Value Assessment in Business Markets: A State-of-Practice Study. Journal of Business-to-Business Marketing, 1(1), 3-29. https://doi.org/10.1300/J033v01n01_02

Avery, J., Fournier, S., \& Wittenbraker, J. (2014). Unlock the Mysteries of Your Customer Relationships. Harvard Business Review, 78), 72-81. https://hbr.org/2014/07/unlock-the-mysteries-of-yourcustomer-relationships\#comment-section

Berger, P., \& Nasr, N. (1998). Customer lifetime value: Marketing models and applications. Journal of $\begin{array}{llll}\text { Interactive } \quad \text { Marketing, } & 12 & \text { 17), }\end{array}$ https://www.sciencedirect.com/science/article/abs/pii/S1094996898702506

Brue, S., \& Grant, R. (2009). Historia del Pensamiento Económico. (8. a ed.) México: CENGAGE Learning.

Carr, R. (1990). La vanguardia al servicio del cliente: 15 calves para satisfacer al cliente. Madrid: Ed. Díaz de Santos.

Fragoso, E. (2006). ¿Son los valores subjetivos u objetivos? Diferenciación entre lo que es un valor en sí y el proceso de valoración. Revista Xihmai, $\quad$ 1(2). http://www.lasallep.edu.mx/xihmai/index.php/xihmai/article/view/43/27

Francés, A. (2006). Estrategia y planes para la empresa: con el cuadro de mando. México: Pearson Educación.

Gale, B. (1994). Manging customer value. Nueva York: The Free Press.

García, I. (2008). CRM: Gestión de la relación con los clientes. Madrid: FC Editorial.

Gil, I., \& Gallarza, M. (2006). Desarrollo de una escala multidimensional para medir el valor percibido de una experiencia de servicio. Revista Española de Investigación de Marketing, 10(2), 25-59. https://dialnet.unirioja.es/servlet/articulo?codigo=2137773

Gilibets, L. (2013). 5 indicadores que no debes olvidar para calcular el valor de tu negocio. Creación de Empresas. [Entrada de blog]. https://www.iebschool.com/blog/calcular-valor-negocio-creacionempresas/ 
Gooodstein, L. \& Butz, H. (2004). Custemer Value: The Linchpin of Organizational Change. Organizational Dynamics, 2オ1), 21-34. https://doi.org/10.1016/S0090-2616(98)90038-9

Gupta, S. (2014). Creating Customer Value. Core Curriculum Readings in Marketing. Boston: Harvard Business Publishing. https://hbsp.harvard.edu/product/8176-PDF-ENG

Gupta, S., \& Lehmann, D. (2001). Customers as assets. Journal of Interactive Marketing, 17(1), 9-24. https://doi.org/10.1002/dir.10045

Gupta, S., Hanssens, D., Hardie, B., Kahn, W. Kumar, V., Lin N. \& Ravishanker, N. (2006). Modeling Customer Lifetime Value. Journal of Service Research, 9(2), 139-155. https://doi.org/10.1177\%2F1094670506293810

Jensen, M. (2008). Value Maximization, Stakeholder Theory, and the Corporate Objective Function. Business Ethics Quarterly, 12(2), 235 -256. https://www.jstor.org/stable/3857812

Joyanes, L., \& Sánchez, A. (2008). Cómo medir el valor del cliente. MK Marketing+Ventas, (238), 64-68. https://dialnet.unirioja.es/servlet/articulo?codigo=2697412

Kaplan, R. \& Narayanan, V., (2001). Measuring and Managing Customer Profitability. Journal of Cost Management, 15(5), 5-15. https://www.hbs.edu/faculty/Pages/item.aspx?num=11052

Kaplan, R., \& Norton, D. (1996). The Balanced Scorecard: Translating Strategy into Action. Boston: Harvard Business School Press.

Kotler, P., Cámara, D., \& Grande I. (1995). Dirección de Marketing. Madrid: Prentice Hall.

Lovelock, C. (1991). Services Marketing: text, cases and readings. USA: Prentice Hall.

Martínez, J., Borja, L. y Carvajal, P. (1999). Fidelizando clientes: detectar y mantener al cliente leal. Barcelona: Gestión 2000.

Marx, K. (1867). El capital: Crítica de la economía política, tomo I, Libro I. El proceso de producción del capital. México: Fondo de Cultura Económica.

Moliner, M., Bigné, J., \& Callarisa, L. (2005). El Valor Percibido en los mercados industriales: Una perspectiva multidimensional. Revista Europea de Dirección y Economía, 15(1), 163-180. https://dialnet.unirioja.es/servlet/articulo?codigo $=1977588$ 
Monroe, K. (1990). Pricing making profitable decisions. New York: McGrawn-Hill.

Ortigosa, M. (2006). El valor del cliente en la incertidumbre. Trabajo presentado en el IV coloquial predoctoral América Latina y Unión Europea: Oportunidades y Desafíos. Asamblea Anual de CLADEA, Montpellier, Francia.

Pons, I. (2012). Customer Lifetime Management (CLM): recopilar la máxima información sobre el cliente [Entrada de blog]. http://www.esencialblog.es/customer-lifetime-management-clm-recopilar-lamaxima-informacion-sobre-el-cliente/

Vargo, S. \& Lusch, L. (2008). Service dominant logic: continuing the evolution. Academy of Marketing Science, (36,) 1-10. https://link.springer.com/article/10.1007/s11747-007-0069-6

Woodruff, R. (1997). Customer value: The next source for competitive advantage. Journal of the Academy of Marketing Science, 25(2), 140-153. https://doi.org/10.1007/BF02894350 